Supporting information

\title{
Biomimetic porous nanofiber-based oil pump for spontaneous oil directional transport and collection
}

Man Jia, Huichao Liu, Guang Yang, Shuo Zhang, Jinglong Yang, Lei Tian* Caizhen $\mathrm{Zhu}^{*}$, and Jian $\mathrm{Xu}$ *

Institute of Low-dimensional Materials Genome Initiative, College of Chemistry and Environmental Engineering, Shenzhen University, Shenzhen, 518060, China.

*Corresponding author:

E-mail: leitian@szu.edu.cn

czzhu@szu.edu.cn

jxu@iccas.ac.cn 


\section{Oil absorption test}

The maximum oil adsorption capacity of PS nanofiber sorbents was carried out using the following method; Approximate $10 \mathrm{mg}$ of fibrous sorbents was placed in a glass beaker filled with $30 \mathrm{~mL}$ of oil. After adsorption for $24 \mathrm{~h}$, the wet sorbent was removed from the oil bath and drained for $1 \mathrm{~min}$. The oil adsorption capacity of the sorbent, Q ( $\left.\mathrm{g} \mathrm{g}^{-1}\right)$, was determined by the equation (1)

$$
Q=\frac{w-w_{0}}{w_{0}}
$$

where $w$ is the total mass of the wet sorbent after $24 \mathrm{~h}$ draining and $w_{0}$ is the mass of the sorbent before adsorption. Each sample was independently measured for at least three times [Macromolecules 2017; 50: 2528-2534.].

2. The image in Figure $1 \mathrm{~b}$ is obtained from https://wenku.baidu.com/view/5a21b417a216147917112821.html 


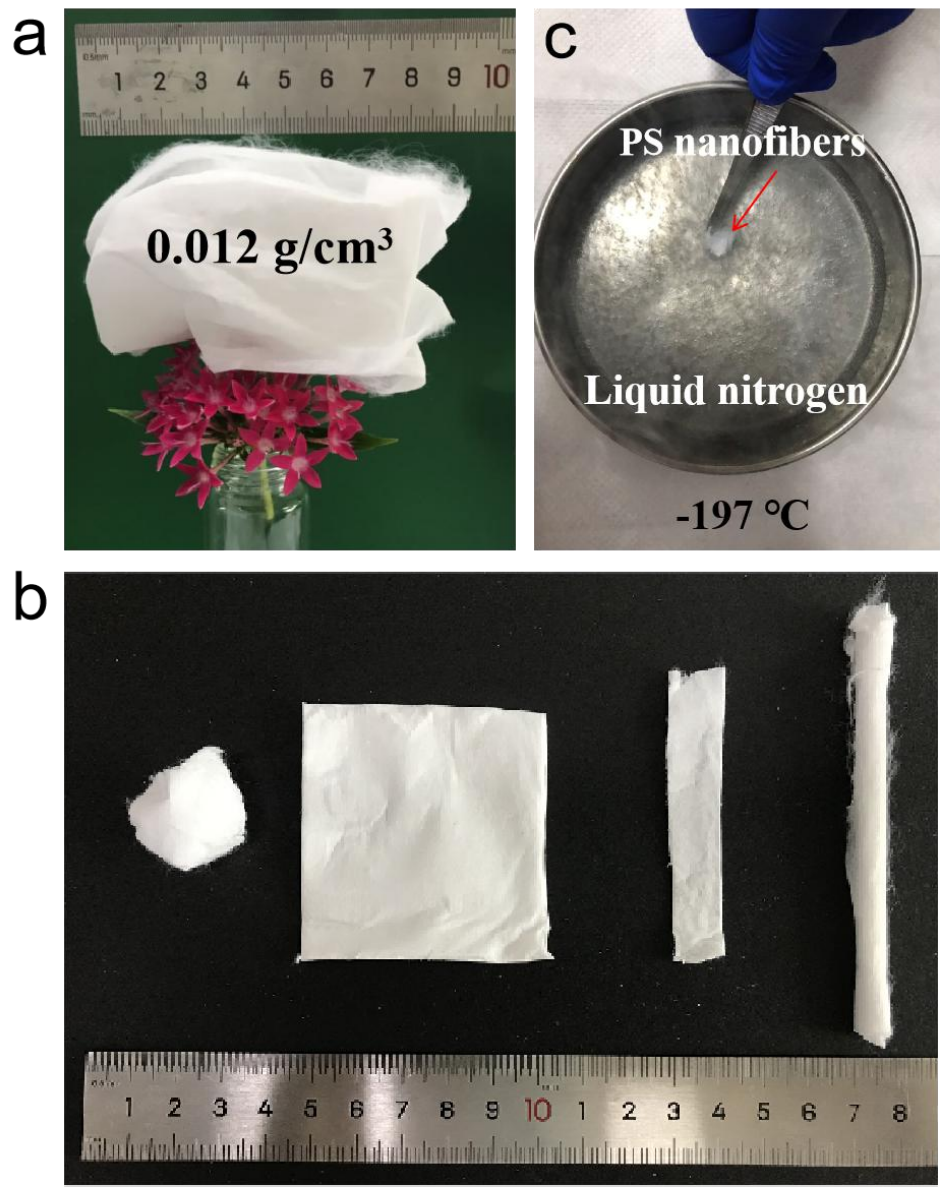

Figure S1. (a) The low apparent density (b) tailor-made manipulation (c) Flexible retention feature in liquid nitrogen of nanoporous fibers. 


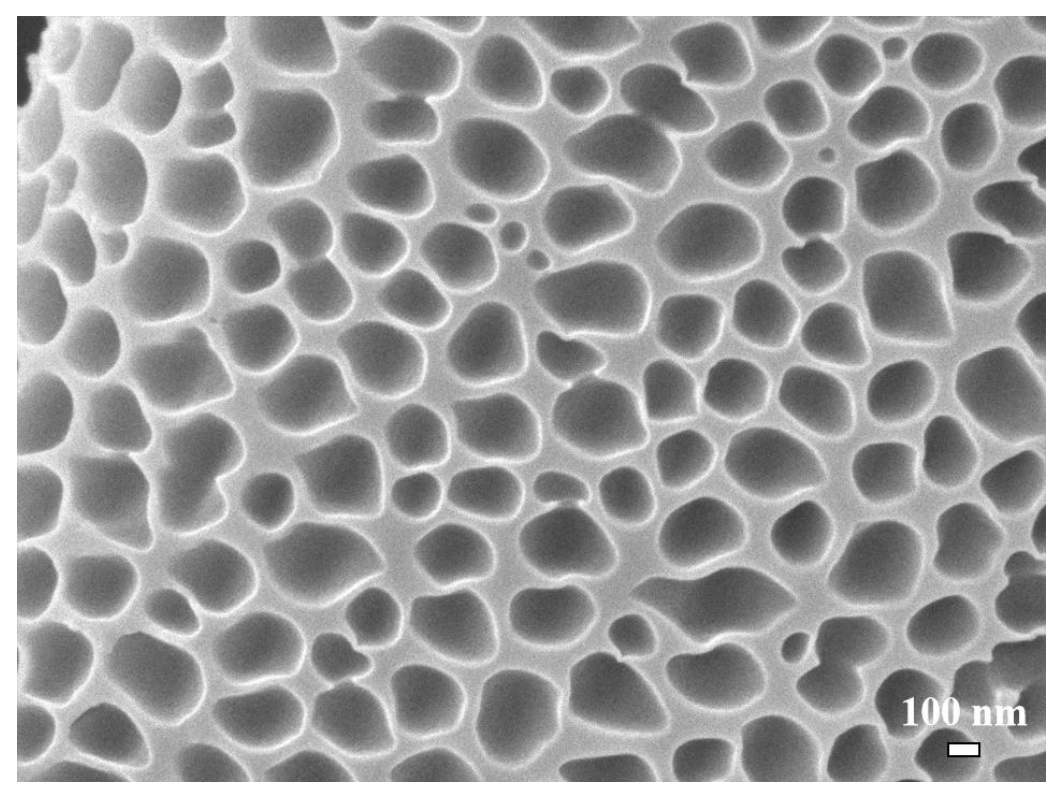

Figure S2. Magnifying SEM image of the uniform pore structure in nanoporous fibers. 

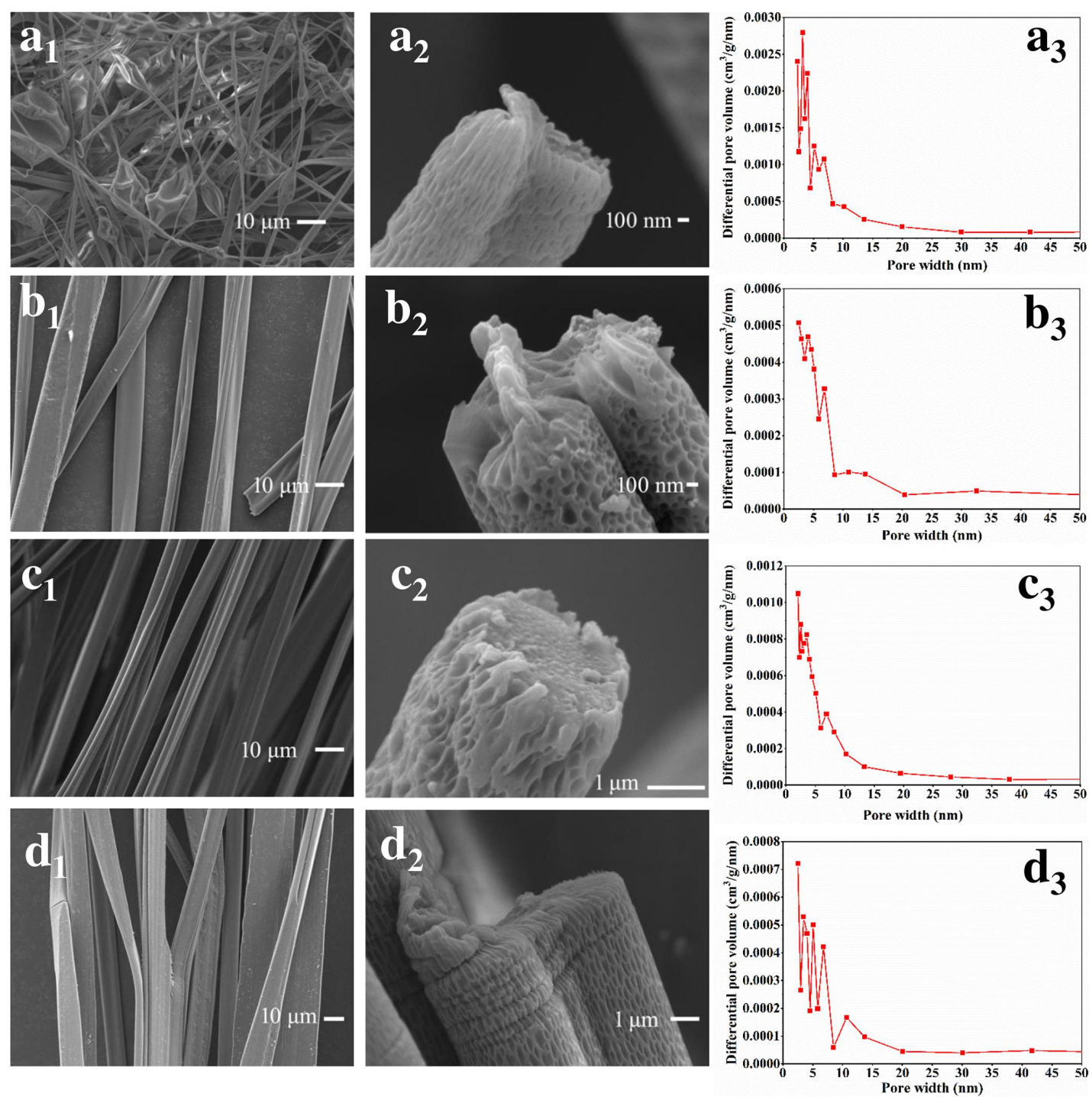

Figure S3. SEM images of the surfaces $\left(a_{1}, b_{1}, c_{1}, d_{1}\right)$, cross sections $\left(a_{2}, b_{2}, c_{2}, d_{2}\right)$ and pore size distribution curves of $\left(a_{3}, b_{3}, c_{3}, d_{3}\right)$ of the PS nanofibers fabricated by the electrospining of $15 \%, 20 \%, 25 \%$ and $30 \mathrm{wt} \%$ PS concentration in THF solution, respectively. Pore size distribution curves were obtained from the desorption branches of the isotherms for nanofibers (T-15, T-20, T-25, T-30) at $77 \mathrm{~K}$. 

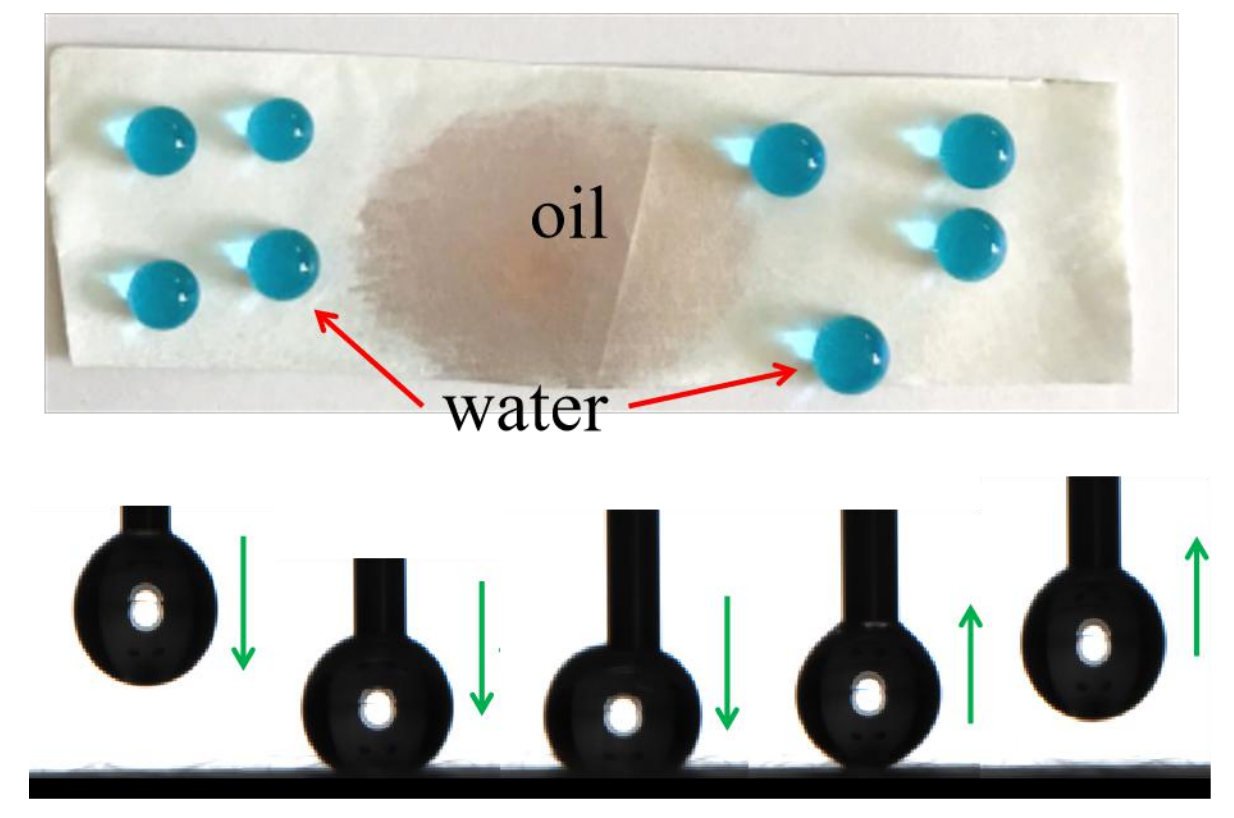

Figure S4. Photograph images of oil droplet and water on nanofibers.

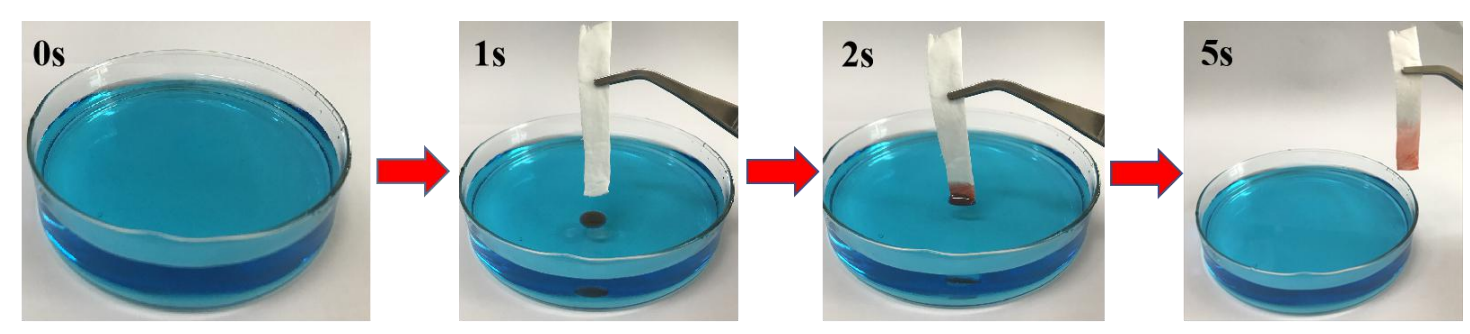

Figure S5. Oil/water separation and absorption capacity of nanofibers T-20-D-2.5. Removal process of n-hexadecane floating on water. 
Table S1 Comparison of the current oil clean-up and the previous devices

\begin{tabular}{cccccc}
\hline Ref. & Pump & Squeezer/ & Heater & Recovery rate & Energy comsuption \\
& \multicolumn{3}{c}{ Centrifuge } & & (KJ/Kg of oil) \\
\hline$[\mathrm{S} 1]$ & Yes & Yes & Yes & $627.8 \% \mathrm{Kg} \mathrm{m}^{-2} \mathrm{~h}^{-1}$ & $54.3 \sim 74.9 \mathrm{Kg} \mathrm{m}^{-2} \mathrm{~h}^{-1}$ \\
{$[\mathrm{~S} 2]$} & Yes & & $580 \% \mathrm{~L} \mathrm{~m}^{-2} \mathrm{~h}^{-1}$ & $\mathrm{E}$ \\
{$[\mathrm{S} 3]$} & Yes & Yes & & $\mathrm{E}$ \\
{$[\mathrm{S} 4]$} & Yes & Yes & & $\mathrm{E}$ \\
{$[\mathrm{S} 5]$} & Yes & & & $\mathrm{E}$ \\
{$[\mathrm{S} 6]$} & Yes & Yes & & $\mathrm{E}$ \\
{$[\mathrm{S} 7]$} & Yes & Yes & & $\mathrm{E}$ \\
This & & & & \\
work & & & & \\
\hline
\end{tabular}

$※$ - Estimated from the data in literatures.

E - Electricity-powered

\section{References}

[S1] He S, Cheng X, Li Z, et al. Green and facile synthesis of sponge-reinforced silica aerogel and its pumping application for oil absorption. J. Mater. Sci. 2016; 51: 292-1301.

[S2] Wang X, Pan Y, Shen C, et al. Facile thermally impacted water-induced phase separation approach for the fabrication of skin-free thermoplastic polyurethane foam and its recyclable counterpart for oil-water separation. Macromol. Rapid Commun. 
2018; 39: 1800635.

[S3] Zhang C, Wu MB, Wu BH, et al. Solar-driven self-heating sponges for highly efficient crude oil spill remediation. J. Mater. Chem. 2018; 6: 8880-8885.

[S4] Jiang ZR, Ge J, Zhou YX, et al. Coating sponge with a hydrophobic porous coordination polymer containing a low-energy $\mathrm{CF}_{3}$-decorated surface for continuous pumping recovery of an oil spill from water. NPG Asia Mater. 2016; 8: e253.

[S5] Liu Y, Shi Q, Hou C, et al. Versatile mechanically strong and highly conductive chemically converted graphene aerogels. Carbon $2017 ; 125: 352-359$.

[S6] Wang Y, Liu X, Lian M, et al. Continuous fabrication of polymer microfiber bundles with interconnected microchannels for oil/water separation. Appl. Mater. Today 2017; 9: 77-81.

[S7] Khosravi M, Azizian S. Synthesis of a novel highly oleophilic and highly hydrophobic sponge for rapid oil spill cleanup. ACS Appl. Mat. Interfaces 2015; 7: 25326-25333. 